\title{
MK615 Suppresses Hypoxia Tolerance by Up-regulation of E-cadherin in Colorectal Cancer Cells With Mutant KRAS
}

\author{
KENSUKE NISHI ${ }^{1,2,3^{*}}$, TOSHIYUKI TSUNODA ${ }^{2,4^{*}}$, YOSHINORI UCHIDA $^{1}$, \\ TAKAYUKI SUETA $^{1}$, MOTOHIRO SAWATSUBASHI $^{5}$, TAKAFUMI YAMANO $^{3}$, \\ YASUKO HASHIGUCHI ${ }^{4}$, ANTHONY SWAIN ${ }^{2}$, SENJI SHIRASAWA ${ }^{2,4}$ and TOSHIFUMI SAKATA ${ }^{1}$ \\ Departments of ${ }^{1}$ Otorhinolaryngology and ${ }^{2}$ Cell Biology, Faculty of Medicine, Fukuoka University, Fukuoka, Japan; \\ ${ }^{3}$ Section of Otolaryngology, Department of Medicine, Fukuoka Dental College, Fukuoka, Japan; \\ ${ }^{4}$ Central Research Institute for Advanced Molecular Medicine, Fukuoka University, Fukuoka, Japan; \\ ${ }^{5}$ Department of Otolaryngology, Fukuoka University Chikushi Hospital, Fukuoka, Japan
}

\begin{abstract}
Background/Aim: The Japanese apricot "Prunus mume" is a traditional Japanese medicine. MK615, a compound extract from Prunus mume has been reported to have anti-tumor effects. Herein, we used $3 D$ floating (3DF) culture to evaluate the anticancer effects of MK615 against human colorectal cancer (CRC) cells that contain mutant (mt) KRAS. Materials and Methods: HKe3 cells exogenously expressing mtKRAS (HKe3-mtKRAS) were treated with MK615 in $3 D F$ cultures. The protein levels of hypoxia-inducible factor 1 (HIF-1) and E-cadherin were quantified by western blotting. Results: MtKRAS enhanced hypoxia tolerance via upregulation of HIF-1. The expression of HIF-1 protein was suppressed by constitutive overexpression of E-cadherin in CRC HCT116 spheroids. MK615 increased the expression of E-cadherin and decreased the expression of HIF-1 in HKe3mtKRAS. These results suggest that MK615 suppresses hypoxia tolerance by up-regulation of E-cadherin in CRC cells with mtKRAS. Conclusion: MK615 exhibits properties useful for the potential treatment of CRC patients with mtKRAS.
\end{abstract}

KRAS is an important downstream molecular switch of cellsurface growth signal receptors such as epidermal growth factor receptor (EGFR), which is closely related to cell proliferation $(1,2)$. Mutations of the KRAS gene occur in

This article is freely accessible online.

*These Authors contributed equally to this study.

Correspondence to: Dr. Toshifumi Sakata, Department of Otorhinolaryngology, Faculty of Medicine, Fukuoka University, 745-1 Jonan-ku, Fukuoka, 814-0180, Japan. Tel: +81 928011011, Fax: +81 928633387, e-mail: sakata@fukuoka-u.ac.jp

Key Words: KRAS, MK615, colon cancer, HIF, E-cadherin, Prunus mume. about $35-40 \%$ of sporadic colorectal cancer (CRC) cases (3$5)$ and are associated with resistance to EGFR inhibitors and overall poor prognosis (6-8). Because drugs directly targeting mutant (mt) KRAS (G13D) have not yet been developed for clinical use (9), we have previously investigated drugs targeting downstream signals of mtKRAS (G13D) (10-12).

MK615 is an extract from the Japanese apricot tree Prunus mume Sieb. Et Zuc (Ume). The exact composition of MK615 has not been reported, but it is known to contain multiple natural compounds including triterpenic acids such as ursolic acid and oleanic acid, phytosterols such as $\beta$ sitosterol, and other active compounds (13). MK615 has been reported to induce several effects such as apoptosis, autophagy, down-regulation of programmed death ligand 1 (PD-L1), and inhibition of Aurora A and B kinases in cancer cells (14-18). However, whether MK615 is effective against cancers expressing mtKRAS remains unclear (9).

Cells grown in three-dimensional floating (3DF) culture reflect the microenvironment of in vivo conditions more accurately than cells cultured in two-dimensional (2D) monolayers (19-21). The inner structure of cancer spheroids is a hypoxic environment, which resembles the state of human solid tumors in vivo (22). The hypoxia inducible factor (HIF)-mediated signaling pathway is important for the survival of cancer cells under hypoxic conditions. HIF-1 plays a pivotal role in hypoxic adaptation (23), which is referred to as hypoxia tolerance. As Kikuchi et al. reported, mtKRAS up-regulates HIF-1 in 2D cultures (24), suggesting that mtKRAS induces hypoxia tolerance via HIF-1 upregulation in $3 \mathrm{DF}$ culture. Recent studies also showed that hypoxia inhibits E-cadherin expression $(25,26)$. E-cadherin is down-regulated by mtKRAS in 2D culture (27), suggesting that mtKRAS may also down-regulate E-cadherin in $3 \mathrm{DF}$ culture, and that the overexpression of E-cadherin modulates hypoxic conditions. 
In this study, we confirmed that mtKRAS enhances hypoxia tolerance via HIF-1 expression, and constitutive expression of E-cadherin suppress hypoxia tolerance in 3DF culture of CRC cells. Notably, MK615 increases E-cadherin expression, which rescues the E-cadherin deficit caused by its mtKRASmediated down-regulation, resulting in suppression of growth and hypoxia tolerance of CRC spheroids.

\section{Materials and Methods}

MK615 preparation. MK615 was kindly provided by AdaBio Co (Takasaki, Gunma, Japan). MK615 was derived from the pressure extraction of apricot fruit juice from which squeezed residue was removed. The juice was then heated and concentrated. Before use, the concentrate was dissolved in water, the $\mathrm{pH}$ was adjusted to 7.0 with $\mathrm{NaOH}$, and the solution was sterilized in an autoclave.

Antibodies and reagents. Anti-E-cadherin, anti-HIF-1 $\alpha$, and antiHIF-1 $\beta$ antibodies were purchased from Cell Signaling Technology (Danvers, MA, USA). Anti-actin antibody was purchased from Sigma-Aldrich (St. Louis, MO, USA).

3DF cell culture. HCT116, HCT116-E-cadherin, HKe3-wtKRAS, and HKe3-mtKRAS cells were maintained as described previously (28, 29). Cells (3,000 per well) were seeded in a round-bottomed 96-well plate with an ultra-low attachment surface (Corning Inc., Corning, NY, USA). Cells were cultured with normal atmospheric oxygen or under hypoxia $\left(\mathrm{O}_{2}: 5 \%\right)$ in a $\mathrm{CO}_{2}$ incubator as described previously $(11,19,28)$. Photomicrographs of cell clusters were taken using a BIOREVO BZ9000 microscope (Keyence, Osaka, Japan).

Three-dimensional imaging of cell clusters. Cell clusters were photographed using the iPhone XR camera system (Apple Inc, Cupertino, CA, USA).

Quantitative real-time reverse-transcriptase polymerase chain reaction ( $R T-q P C R)$. RT-qPCR was performed as previously described (30). The sequences of the primers were as follows: Ecadherin, 5'-ATGAGTGTCCCCCGGTATCT-3' (sense) and 5'TCAGGGAGCTCAGACTAGCAG-3' (anti-sense); $\beta$-Actin, 5'CACCAACTGGGACGACAT-3' (sense) and 5'-ACAGCCTGGA TAGCAACG-3' (anti-sense).

Western blotting. Cells were lysed in RIPA buffer ( $50 \mathrm{mM}$ Tris-HCl, $\mathrm{pH} 7.5,150 \mathrm{mM} \mathrm{NaCl}, 1 \% \mathrm{NP} 40,0.5 \%$ sodium deoxycholate, $0.1 \%$ sodium dodecyl sulfate) containing protease inhibitor cocktail (Roche, Basel, Switzerland) and were subjected to immunoblotting as described previously (31). Quantitative analysis of immunoblots was performed using the ImageJ software (National Institutes of Health, Bethesda, MD, USA).

Retroviral production and generation of stable cell lines. The cDNA for human E-cadherin was inserted into a pMSCVpuro vector. Retroviruses were produced by transfection of the retroviral vectors as described previously (28). HCT116 cells in six-well plates were infected with the viruses by centrifugation for $2 \mathrm{~h}$ at $32^{\circ} \mathrm{C}$ and 2000 $\times g$. Approximately $48 \mathrm{~h}$ after infection, the cells were treated with 2 $\mu \mathrm{g} / \mathrm{ml}$ hygromycin (WAKO, Osaka, Japan) for one week to establish HCT116-derived cells stably expressing E-cadherin.
Immunofluorescence labeling. Immunofluorescence labeling using E-cadherin (Cell Signaling Technology) was performed as previously described $(19,32)$. Cells were examined using a TCS SP5 laser-scanning confocal microscope (Leica Microsystems, Wetzlar, Germany).

Statistical analyses in cell culture experiments. Statistical analyses were performed using unpaired two-tailed Student's $t$-tests. All $p$ values below 0.05 were considered to be statistically significant.

\section{Results}

MtKRAS enhances hypoxia tolerance in CRC cells. To confirm whether hypoxia tolerance is affected by mtKRAS, we compared cell growth between Hke3-wild type (wt)KRAS and HKe3-mtKRAS cells under normoxia and hypoxia in 3DF culture. The growth rate of HKe3-wtKRAS spheroids under hypoxic conditions was 2.1-fold lower than that under normal oxygen conditions at day 12 of $3 \mathrm{DF}$ culture. No significant differences in growth rates were observed between normoxia and hypoxia conditions in HKe3-mtKRAS spheroids at day 12 (Figure 1A).

HIF-1 is a key regulator of hypoxia tolerance. To identify whether mtKRAS is responsible for hypoxia tolerance, we examined the protein levels of HIF- $1 \alpha$ and HIF- $1 \beta$ using Hke3-wtKRAS and HKe3-mtKRAS cells under normoxia or hypoxia for 12 days in $3 \mathrm{DF}$ culture. The protein levels of HIF- $1 \alpha$ and HIF-1 $\beta$ in HKe3-mtKRAS were 1.2-fold and 2.2-fold higher, respectively, compared to those of HKe3wtKRAS (Figure $1 \mathrm{~B}$ and $\mathrm{C}$ ). These results suggest that mtKRAS enhances hypoxia tolerance via up-regulation of HIF-1 in CRC spheroids.

E-cadherin suppressed hypoxia tolerance of colorectal cancer cells in 3DF culture. To confirm whether E-cadherin expression is suppressed by mtKRAS in $3 \mathrm{DF}$ culture, we examined the mRNA and protein levels of E-cadherin using Hke3-wtKRAS and HKe3-mtKRAS spheroids. At day 2 of 3DF culture, mRNA levels of the E-cadherin gene of HKe3-mtKRAS spheroids were 5.7-fold lower compared to those in HKe3-wtKRAS spheroids (Figure 2A). Furthermore, E-cadherin mRNA levels of the spheroids at day 7 were 2.3 -fold lower compared to those of the spheroids at day 2 in HKe3-mtKRAS (Figure 2B). Protein levels of E-cadherin in HKe3-mtKRAS spheroids were also lower compared to those in HKe3-wtKRAS spheroids (Figure 2C). These results suggest that mtKRAS suppresses the expression levels of E-cadherin, and that E-cadherin expression decreases as spheroids grow in 3DF culture.

To address the influence of E-cadherin on hypoxia tolerance, we established human colorectal HCT116 cells that stably express E-cadherin (HCT116-E-Cadherin) (Figure 2D and E). The areas of the HCT116-E-cadherin spheroids were 1.2 and 1.6-fold lower compared to those of control HCT116 spheroids at day 8 under normoxia and hypoxia, respectively (Figure $2 \mathrm{~F}$ 
A

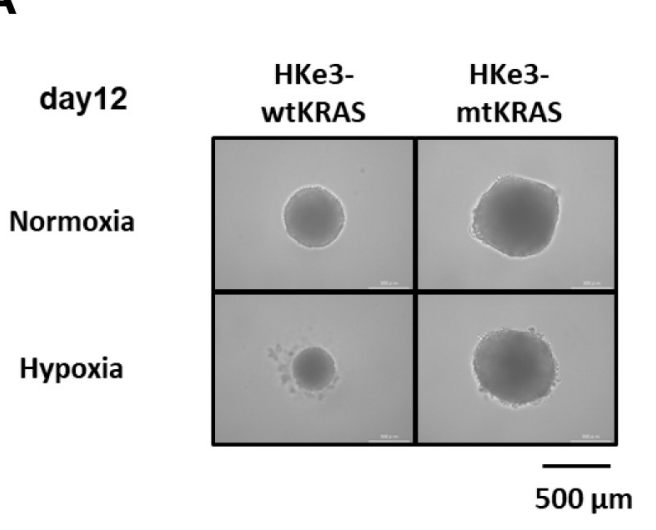

B

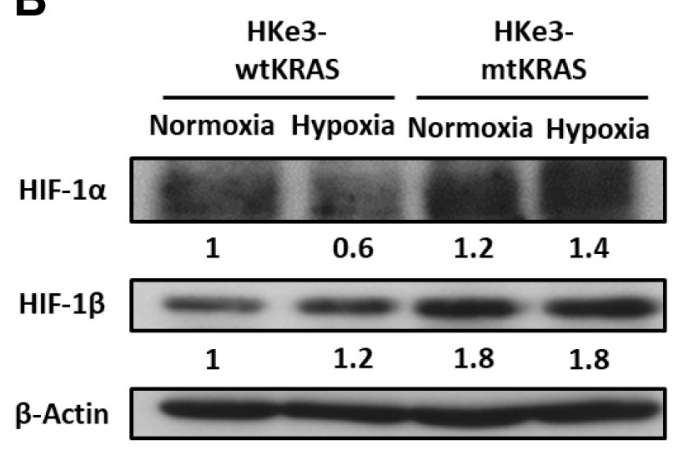

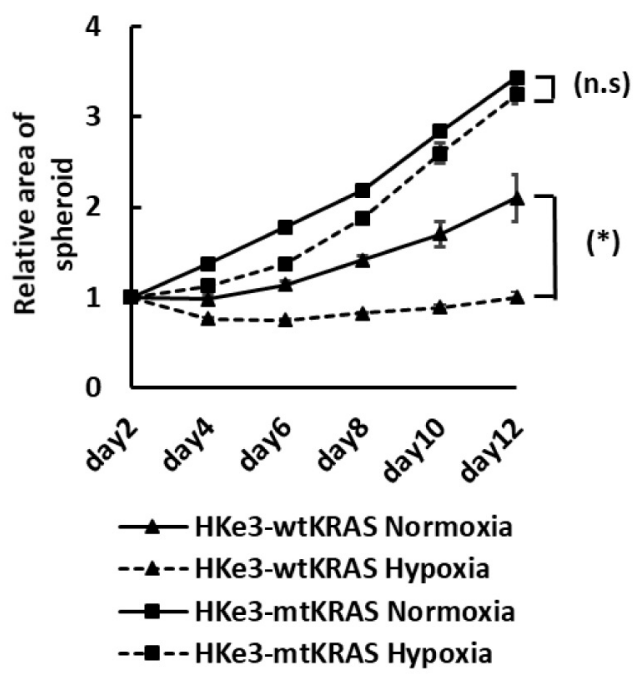

C

Normoxia

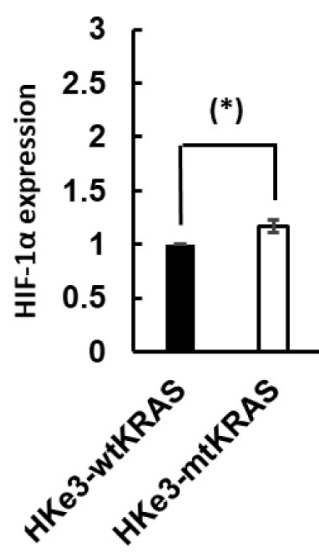

(*)

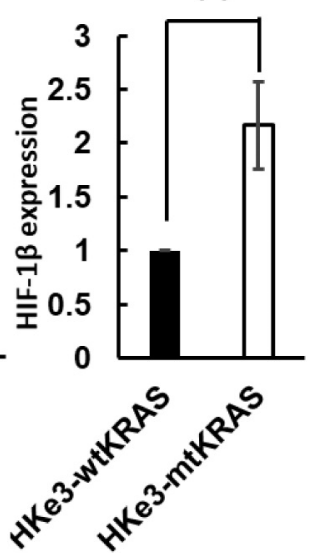

Figure 1. Mutant (mt)KRAS enhances hypoxia tolerance in 3DF culture. (A) Left panel: Growth of HKe3-wild type (wt)KRAS and HKe3-mtKRAS spheroids under normoxia and hypoxia at day 12. Right panel: Growth rate of HKe3-wtKRAS and HKe3-mtKRAS spheroids under normoxia and hypoxia. (B) Left panel: Expression of hypoxia-inducible factor (HIF)-1 protein in HKe3-wtKRAS and HKe3-mtKRAS spheroids under normoxia and hypoxia at day 12. Levels of proteins were quantified by densitometry and normalized to actin levels. Values represent the relative ratio of each sample to levels in HKe3-wtKRAS spheroids grown under normoxia. Right panel: Relative expression of HIF-1 protein ratio to levels in HKe3-wtKRAS spheroids under normoxia are shown. n.s.: Not significantly different; significantly different at $* p<0.05$. Data are representative of three biological replicates.

and G). Surprisingly, HCT116-E-cadherin spheroids collapsed spontaneously at day 8 under hypoxia (Figure 2F), suggesting a loss of hypoxic tolerance. Furthermore, we showed that the expression of HIF- $1 \alpha$ and $\beta$ decreased in HCT116-E-cadherin spheroids in 3DF culture (Figure $2 \mathrm{H}$ ). These results suggested that E-cadherin suppresses hypoxia tolerance via HIF-1 downregulation in $3 \mathrm{DF}$ culture.

MK615 suppresses the ability of spheroid formation and proliferation in CRC cells with mtKRAS. To address the effects of MK615 against CRC cells with mtKRAS, HKe3-mtKRAS cells were treated with MK615 in 3DF culture. Areas of the HKe3-mtKRAS spheroids at day 11 were 2.3-fold higher compared to those at day 1. By contrast, HKe3-mtKRAS cells treated with MK615 did not aggregate at day 1, and the area of the spheroids subsequently decreased. The areas of the HKe3-mtKRAS spheroids with MK615 at day 11 were 1.5fold lower compared to those at day 1 (Figure 3A and B). While HKe3-mtKRAS cells produced round-shaped spheroids $(\mathrm{A} / \mathrm{B}=1.0), \mathrm{HKe} 3-\mathrm{mtKRAS}$ cells treated with MK615 


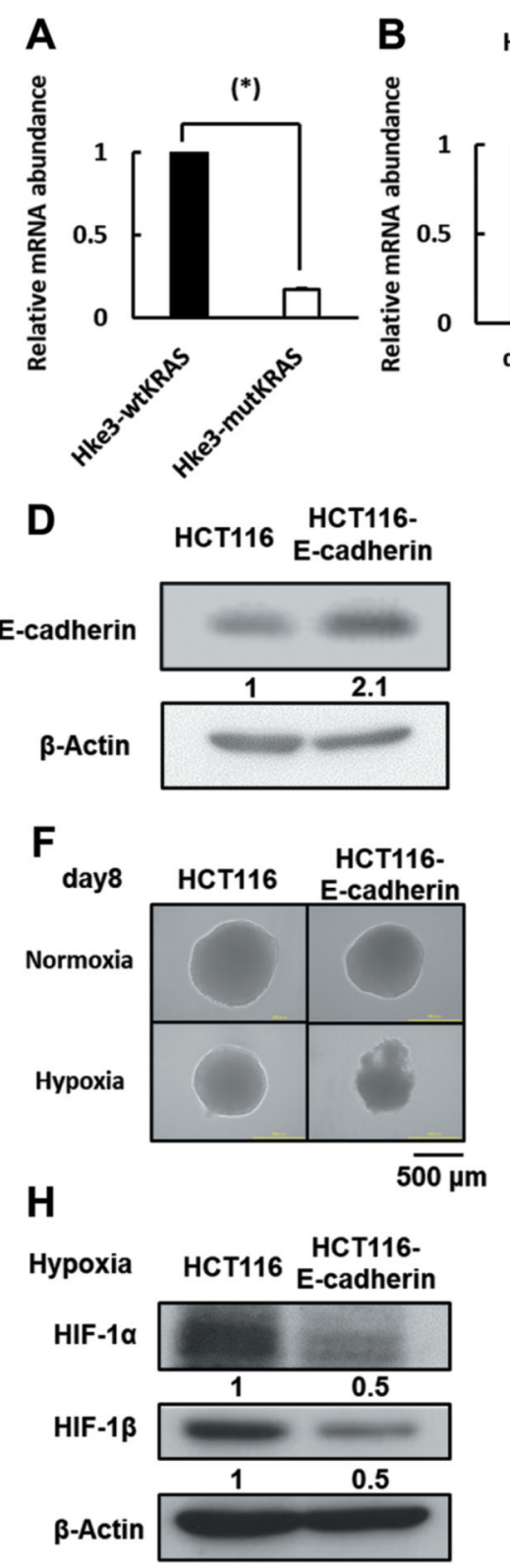

E

HCT116

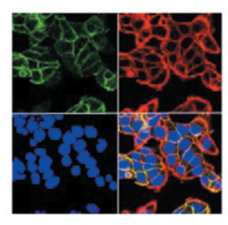

$\mathbf{G}$

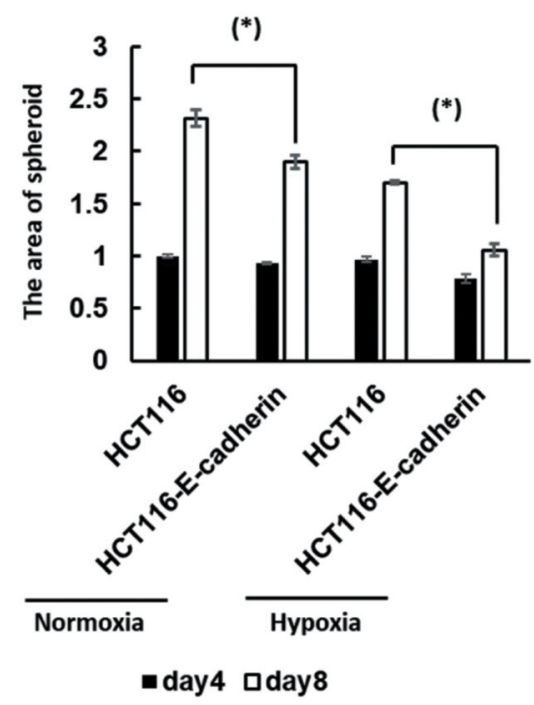

Figure 2. Constitutive overexpression of E-cadherin on HCT116 cells suppressed hypoxia tolerance in 3DF culture. (A) Quantitative RT-PCR analysis of Ecadherin gene in human colorectal cancer HKe3 cells stably expressing wild type (wt)KRAS (HKe3-wtKRAS) or mutant (mt)KRAS (HKe3-mtKRAS) at day 2 in $3 D F$ culture. The $m R N A$ expression levels of E-cadherin were normalized against those of $\beta$-actin. Results are shown as the values relative to HKe3wtKRAS. Significantly different at $* p<0.05$. Data are average of three biological replicates. (B) RT-qPCR analysis of the E-cadherin gene in HKe3-mtKRAS cells at day 2 and day 7 in $3 D F$ culture. The mRNA expression levels of E-cadherin were normalized against those of $\beta$-actin. Results are shown as the values relative to HKe3-mtKRAS at day 2. *Significantly different at $p<0.05$. Data are the average of three biological replicates. (C) Expression of E-cadherin protein in HKe3-wtKRAS cells and HKe3-mtKRAS cells at day 2 in 3DF culture. Levels of proteins were quantified by densitometry and normalized to $\beta$ actin levels. Values represent the relative ratio to levels in HKe3-wtKRAS cells grown. Data are representative of three biological replicates. (D) Expression of E-cadherin protein in wild-type HCT116 cells and HCT116 cells stably expressing E-cadherin (HCT116-E-cadherin) grown in 3D culture. $\beta$-actin was used as a loading control. Protein levels were quantified by densitometry and normalized to $\beta$-actin levels. Values represent the relative ratios to levels in wild-type HCT116 cells. Data are representative of three biological replicates. (E) Signals for antibody to E-cadherin in wild-type HCT116 cells and HCT116E-cadherin cells grown in 2D culture. E-cadherin, green; phalloidin-labelled F-actin, red; 4',6-diamidino-2-phenylindole (DAPI)-labelled nuclei, blue. (F) The growth of HKe3 spheroids and HCT116-E-cadherin spheroids under normoxia and hypoxia at day 8. Data are representative of three biological replicates. (G) Areas of wild-type HCT116 or HCT116-E-cadherin spheroids under normoxia and hypoxia at day 4 and day 8 . Values represent the relative ratios to levels in HCT116 spheroids grown at day 4. Significantly different at ${ }^{*} p<0.05$. Data are the average of three biological replicates. (H) Protein expression of HIF in wild-type HCT16 spheroids and HCT116-E-cadherin spheroids under hypoxia at day 8. Protein levels were quantified by densitometry and normalized to $\beta$-actin levels. Values represent the relative ratios to levels in wild-type HCT116 spheroids. Data are representative of three biological replicates. 
A

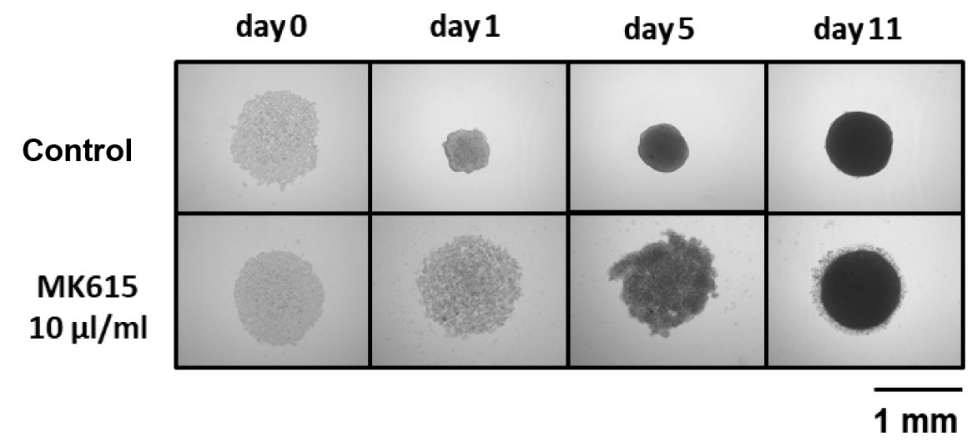

B

$(*)$

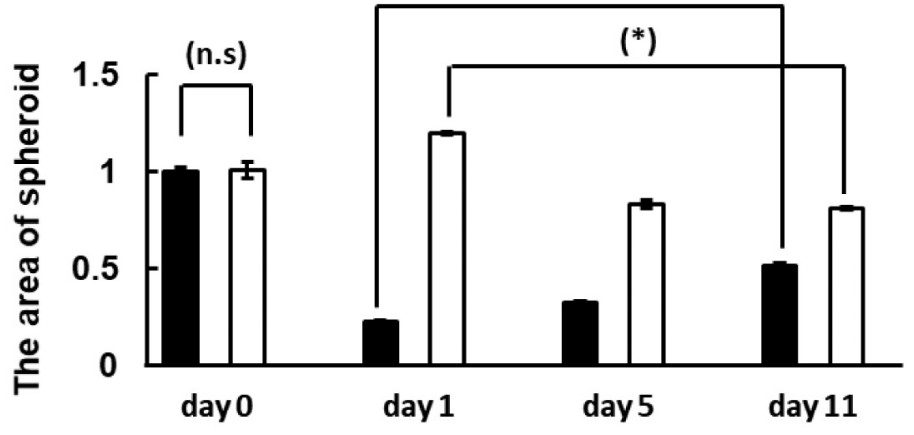

C
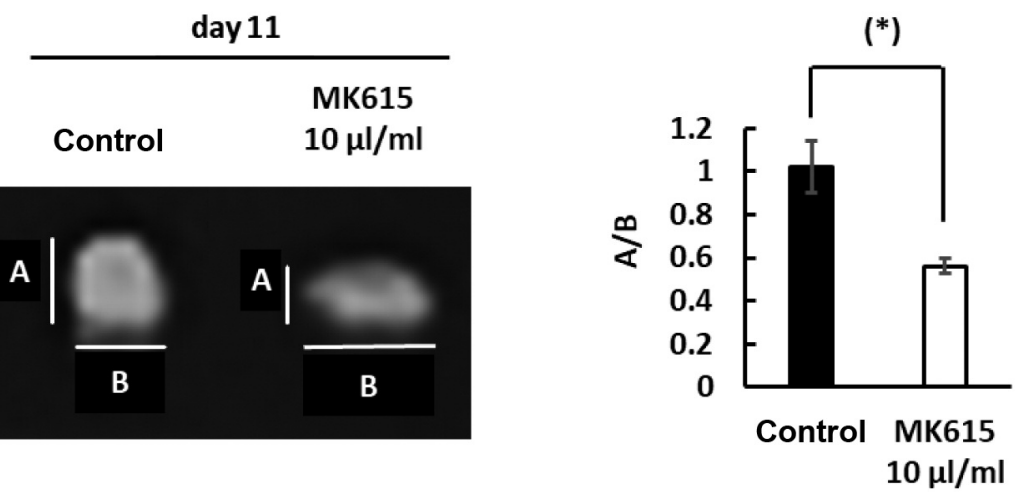

D

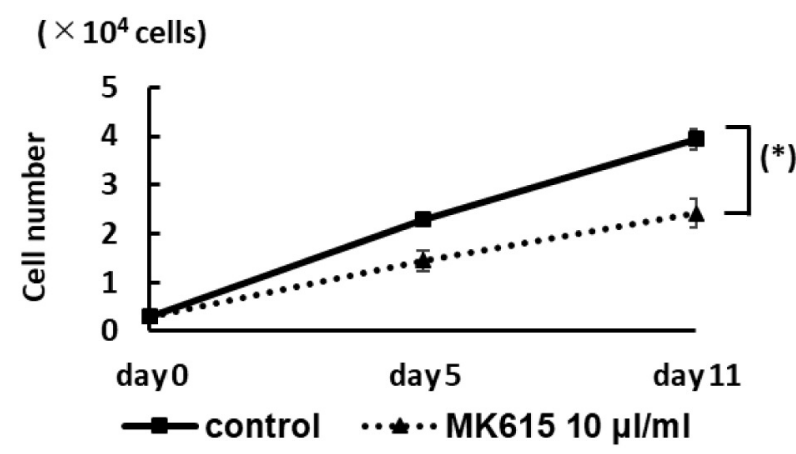

Figure 3. MK615 suppresses the ability of spheroid formation and proliferation in HKe3-mutant (mt)KRAS spheroids. (A) Cell growth of HKe3mtKRAS spheroids with or without MK615 treatment at days 0, 1, 5, and 11 in 3D floating culture. (B) The area of HKe3-mtKRAS spheroids with or without MK615 treatment at day 0, 1, 5, and 11. Values represent the relative ratios to levels in HKe3-mtKRAS spheroids without MK615 treatment grown at day 0. (C) Structure of HKe3-mtKRAS spheroids with or without MK615 treatment at day 11. A, thickness; B, diameter. (D) Cell proliferation of HKe3-mtKRAS spheroids with or without MK615 treatment. For all panels, significantly different at $* p<0.05$. Data are the average of three independent experiments. 
A

HIF-1 $\alpha$

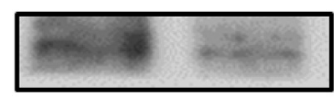

1

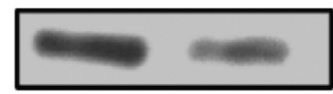

1

Normoxia

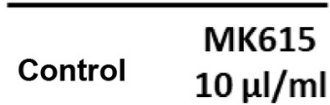

HIF-1 $\beta$

B-Actin

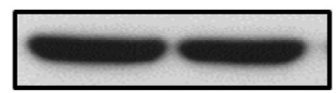

Hypoxia

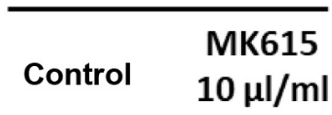

HIF-1 $\alpha$

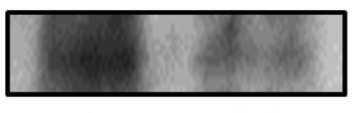

1

0.6

HIF-1 $\beta$

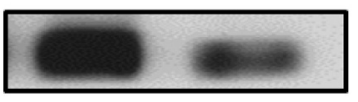

$\beta$-Actin

0.5

1

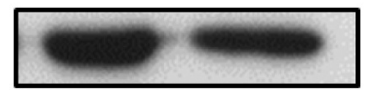

B

MK615 ( $\mu \mathrm{l} / \mathrm{ml})$

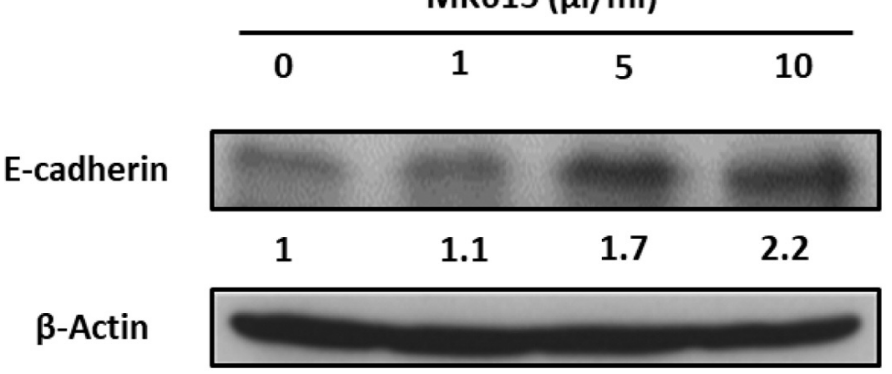

Figure 4. MK615 suppressed the expression levels of hypoxia-inducible factor (HIF)-1 $\alpha$ and $\beta$ and accelerated the expression levels of E-cadherin in HKe3-mutant (mt)KRAS spheroids. (A) Expression of HIF protein in HKe3-mtKRAS spheroids with MK615 under normoxia or hypoxia at day 11. (B) Expression of E-cadherin protein in HKe3-mtKRAS spheroids with MK615 at day 11. For both panels, $\beta$-actin was used as a loading control; protein levels were quantified by densitometry and normalized to those of $\beta$-actin. Values represent the relative ratios to levels in HKe 3 -mtKRAS spheroids without MK615. Data are representative of three biological replicates.

produced discoid cell clusters $(\mathrm{A} / \mathrm{B}=0.6)$ (Figure 3C). These results suggest that MK615 suppresses cell aggregation and the ability of spheroid formation (spheroidgenesis).

To address the effects of MK615 on cell proliferation, the number of cells was counted from single spheroids. In HKe3-mtKRAS spheroids treated with MK615, the number of cells was 1.63-fold lower compared to those of HKe3mtKRAS spheroids at day 11 (Figure 3D), suggesting that MK615 suppresses cell proliferation in HKe3-mtKRAS spheroids.

MK615 suppressed the expression levels of HIF-1 $\alpha$ and $\beta$ and up-regulated the expression levels of E-cadherin in CRC cells with mtKRAS in $3 D F$ culture. To determine whether MK615 affects hypoxia tolerance regulated by the HIF-1 pathway in HKe3-mtKRAS spheroids, we examined the expression of HIF- $1 \alpha$ and HIF-1 $\beta$ proteins in HKe3mtKRAS spheroids treated with MK615 at day 11. Regardless of oxygen concentration, MK615 suppressed expression of both HIF- $1 \alpha$ and HIF- $1 \beta$ proteins (Figure 4A). These results suggested that MK615 suppresses the HIF-1 pathway enhanced by mtKRAS in 3DF culture. Furthermore, MK615 accelerated the protein levels of E-cadherin downregulated by mtKRAS in a dose-dependent manner in HKe3mtKRAS at day 11 (Figure 4B). These results suggest that MK615 suppresses the expression levels of HIF-1 and induces the expression levels of E-cadherin in HKe3mtKRAS spheroids.

\section{Discussion}

HIF-1, a key regulator of hypoxia tolerance, is a heterodimeric protein consisting of two subunits, HIF- $1 \alpha$ and HIF- $1 \beta$. Under hypoxic conditions, HIF- $1 \alpha$ is stabilized and binds to HIF- $1 \beta$, thus forming a complex of HIF- $1 \alpha$ with HIF- $1 \beta$. This complex works as a transcription factor to regulate numerous target genes involved in survival, proliferation, energy metabolism, apoptosis, tumor vascularization, and metastasis $(33,34)$. We 
herein demonstrated that the protein levels of HIF-1 $\alpha$ and HIF$1 \beta$ within HKe3-mtKRAS spheroids were higher compared to those of HKe3-wtKRAS spheroids (Figure 1B). Our results are consistent with the finding that the Ras oncogene regulates HIF-1 $\alpha$ expression in 2D culture (24). No significant differences in the growth rates of the spheroids were observed under normoxia and hypoxia in HKe3-mtKRAS spheroids (Figure 1A), suggesting that mtKRAS induces hypoxia tolerance via up-regulation of HIF-1 in CRC spheroids under hypoxic conditions.

We also demonstrated that mtKRAS down-regulates Ecadherin at the transcriptional level in 3DF culture (Figure 2A). Our findings are partially supported by a previous report that KRAS represses E-cadherin expression by inducing hypermethylation of the E-cadherin promoter (27). We also found that mRNA expression of E-cadherin decreases as HKe3-mtKRAS spheroids grow (Figure 2B). Riffle et al. reported that the increased volume of spheroids is associated with the increased area of hypoxia (35). Our results together with these reports suggest the link between hypoxia and Ecadherin. Interestingly, we demonstrated that constitutive expression of E-cadherin reduces HIF-1 expression in HCT116 spheroids (Figure 2D-H). These results suggest that E-cadherin expression suppresses hypoxia tolerance in $3 \mathrm{DF}$ culture.

The addition of MK615 notably suppressed the ability of spheroid formation and proliferation in HKe3-mtKRAS spheroids (Figure 3C and D). Furthermore, MK615 upregulated the expression levels of E-cadherin and suppressed the expression levels of HIF- $1 \alpha$ and $\beta$ in HKe3-mtKRAS spheroids (Figure 4A and B). E-cadherin expression is reported to abrogate spheroidogenesis in ovarian cancer cells (36). Furthermore, Depping et al. reported that the cell growth is inhibited and tumor spheroids are changed from spherical to irregular forms due to a decrease in HIF- $1 \alpha$ protein expression in 3D culture (37). Our results together with the previous reports suggest that that MK615 inhibits hypoxia tolerance by down-regulation of HIF-1 via upregulation of E-cadherin in CRC spheroids with mtKRAS. Although further in vitro and in vivo studies are needed to address the efficacy of MK615 for tumor suppression, the present study demonstrates that MK615 has possibilities to be a promising drug for cancer therapy.

\section{Conflicts of Interest}

The Authors declare that they have no competing interests.

\section{Authors' Contributions}

K.N. and T.T. designed the study, performed the experiments, analyzed the data and wrote the paper; Y.U., T.S., Y.H. and A.S. collected the data; M.S., T.Y., S.S., and T.S gave technical support and conceptual advice.

\section{Acknowledgements}

This work was supported by a Grant-in-Aid for research from the Ministry of Education, Culture, Sports, Science, and Technology (MEXT) of Japan and the Fukuoka Foundation for Sound Health Cancer Research Fund. The Authors thank Hao Luo and Yumiko Hirose for their technical assistance.

\section{References}

1 Khosravi-Far $\mathrm{R}$ and Der $\mathrm{CJ}$ : The ras signal transduction pathway. Cancer Metastasis Rev 13(1): 67-89, 1994. PMID: 8143346. DOI: $10.1007 /$ bf00690419

2 Fearon ER and Vogelstein B: A genetic model for colorectal tumorigenesis. Cell 61(5): 759-767, 1990. PMID: 2188735. DOI: 10.1016/0092-8674(90)90186-i

3 Chang YY, Lin PC, Lin HH, Lin JK, Chen WS, Jiang JK, Yang SH, Liang WY and Chang SC: Mutation spectra of ras gene family in colorectal cancer. Am J Surg 212(3): 537-544 e533, 2016. PMID: 27394063. DOI: 10.1016/j.amjsurg.2016.02.013

4 Eklof V, Wikberg ML, Edin S, Dahlin AM, Jonsson BA, Oberg A, Rutegard J and Palmqvist R: The prognostic role of kras, braf, pik3ca and pten in colorectal cancer. Br J Cancer 108(10): 2153-2163, 2013. PMID: 23660947. DOI: 10.1038/bjc.2013.212

5 Neumann J, Zeindl-Eberhart E, Kirchner T and Jung A: Frequency and type of kras mutations in routine diagnostic analysis of metastatic colorectal cancer. Pathol Res Pract 205(12): 858-862, 2009. PMID: 19679400. DOI: 10.1016/j.prp.2009.07.010

6 Garcia-Rostan G, Zhao H, Camp RL, Pollan M, Herrero A, Pardo J, Wu R, Carcangiu ML, Costa J and Tallini G: Ras mutations are associated with aggressive tumor phenotypes and poor prognosis in thyroid cancer. J Clin Oncol 21(17): 32263235, 2003. PMID: 12947056. DOI: 10.1200/jco.2003.10.130

7 Castagnola P and Giaretti W: Mutant kras, chromosomal instability and prognosis in colorectal cancer. Biochim Biophys Acta 1756(2): 115-125, 2005. PMID: 16112461. DOI: 10.1016/j.bbcan.2005.06.003

8 Baynes RD and Gansert J: Kras mutational status as a predictor of epidermal growth factor receptor inhibitor efficacy in colorectal cancer. Am J Ther 16(6): 554-561, 2009. PMID: 19352138. DOI: 10.1097/MJT.0b013e318199fa17

9 Karapetis CS, Khambata-Ford S, Jonker DJ, O'Callaghan CJ, Tu D, Tebbutt NC, Simes RJ, Chalchal H, Shapiro JD, Robitaille S, Price TJ, Shepherd L, Au HJ, Langer C, Moore MJ and Zalcberg JR: K-ras mutations and benefit from cetuximab in advanced colorectal cancer. N Engl J Med 359(17): 1757-1765, 2008. PMID: 18946061. DOI: 10.1056/NEJMoa0804385

10 Tsunoda T, Ota T, Fujimoto T. DOI K, Tanaka Y, Yoshida Y, Ogawa M, Matsuzaki H, Hamabashiri M, Tyson DR, Kuroki M, Miyamoto S and Shirasawa S: Inhibition of phosphodiesterase4 (pde4) activity triggers luminal apoptosis and akt dephosphorylation in a 3-d colonic-crypt model. Mol Cancer 11: 46, 2012. PMID: 22830422. DOI: 10.1186/1476-4598-11-46

11 Nishi K, Luo H, Ishikura S. DOI K, Iwaihara Y, Wills L, Baillie GS, Sakata T, Shirasawa S and Tsunoda T: Apremilast induces apoptosis of human colorectal cancer cells with mutant kras. Anticancer Res 37(7): 3833-3839, 2017. PMID: 28668883. DOI: 10.21873/anticanres. 11762

12 Luo H, Nishi K, Ishikura S, Swain A, Morishige N, Yazaki R, Ohshima T, Shirasawa S and Tsunoda T: Growth suppression of human colorectal cancer cells with mutated kras by 3 -deaza- 
cytarabine in 3d floating culture. Anticancer Res 38(7): 42474256, 2018. PMID: 29970558. DOI: 10.21873/anticanres.12721

13 Bailly C: Anticancer properties of prunus mume extracts (chinese plum, japanese apricot). J Ethnopharmacol 246: 112215, 2020. PMID: 31491438. DOI: 10.1016/j.jep.2019.112215

14 Yanaki M, Kobayashi M, Aruga A, Nomura M and Ozaki M: In vivo antitumor effects of mk615 led by pd-11 downregulation. Integr Cancer Ther 17(3): 646-653, 2018. PMID: 29665734. DOI: $10.1177 / 1534735418766403$

15 Inoue M, Honma Y, Urano T and Suzumiya J: Japanese apricot extract (mk615) potentiates bendamustine-induced apoptosis via impairment of the DNA damage response in lymphoma cells. Oncol Lett 14(1): 792-800, 2017. PMID: 28693235. DOI: 10.3892/ol.2017.6219

16 Nakagawa A, Sawada T, Okada T, Ohsawa T, Adachi M and Kubota K: New antineoplastic agent, mk615, from ume (a variety of) japanese apricot inhibits growth of breast cancer cells in vitro. Breast J 13(1): 44-49, 2007. PMID: 17214792. DOI: 10.1111/j.1524-4741.2006.00361.x

17 Mori S, Sawada T, Okada T, Ohsawa T, Adachi M and Keiichi K: New anti-proliferative agent, mk615, from japanese apricot "prunus mume" induces striking autophagy in colon cancer cells in vitro. World J Gastroenterol 13(48): 6512-6517, 2007. PMID: 18161921. DOI: $10.3748 /$ wjg.v13.i48.6512

18 Okada T, Sawada T, Osawa T, Adachi M and Kubota K: A novel anti-cancer substance, mk615, from ume, a variety of japanese apricot, inhibits growth of hepatocellular carcinoma cells by suppressing aurora a kinase activity. Hepatogastroenterology 54(78): 1770-1774, 2007. PMID: 18019715.

19 Tsunoda T, Takashima Y, Fujimoto T, Koyanagi M, Yoshida Y. DOI K, Tanaka Y, Kuroki M, Sasazuki T and Shirasawa S: Three-dimensionally specific inhibition of DNA repair-related genes by activated kras in colon crypt model. Neoplasia 12(5): 397-404, 2010. PMID: 20454511. DOI: 10.1593/neo.10170

20 Takagi A, Watanabe M, Ishii Y, Morita J, Hirokawa Y, Matsuzaki $\mathrm{T}$ and Shiraishi T: Three-dimensional cellular spheroid formation provides human prostate tumor cells with tissue-like features. Anticancer Res 27(1A): 45-53, 2007. PMID: 17352215.

21 Dolznig H, Rupp C, Puri C, Haslinger C, Schweifer N, Wieser E, Kerjaschki D and Garin-Chesa P: Modeling colon adenocarcinomas in vitro a 3D co-culture system induces cancerrelevant pathways upon tumor cell and stromal fibroblast interaction. Am J Pathol 179(1): 487-501, 2011. PMID: 21703426. DOI: 10.1016/j.ajpath.2011.03.015

22 Akamine T, Morodomi Y, Harada Y, Teraishi K, Tagawa T, Okamoto $\mathrm{T}$, Maehara Y and Yonemitsu Y: Mir-3148 is a novel onco-microrna that potentiates tumor growth in vivo. Anticancer Res 38(10): 56935701, 2018. PMID: 30275189. DOI: 10.21873/anticanres.12906

23 Yun CW, Lee JH and Lee SH: Hypoxia-induced pgc-1alpha regulates mitochondrial function and tumorigenesis of colorectal cancer cells. Anticancer Res 39(9): 4865-4876, 2019. PMID: 31519589. DOI: 10.21873/anticanres.13672

24 Kikuchi H, Pino MS, Zeng M, Shirasawa S and Chung DC: Oncogenic kras and braf differentially regulate hypoxia-inducible factor-1alpha and -2alpha in colon cancer. Cancer Res 69(21): 84998506, 2009. PMID: 19843849. DOI: 10.1158/0008-5472.can-092213

25 Imai T, Horiuchi A, Wang C, Oka K, Ohira S, Nikaido T and Konishi I: Hypoxia attenuates the expression of e-cadherin via upregulation of snail in ovarian carcinoma cells. Am J Pathol 163(4): 1437-1447, 2003. PMID: 14507651. DOI: 10.1016/s00029440(10)63501-8
26 Liu KH, Tsai YT, Chin SY, Lee WR, Chen YC and Shen SC: Hypoxia stimulates the epithelial-to-mesenchymal transition in lung cancer cells through accumulation of nuclear beta-catenin. Anticancer Res 38(11): 6299-6308, 2018. PMID: 30396950. DOI: 10.21873 /anticanres.12986

27 Kwon O, Jeong SJ, Kim SO, He L, Lee HG, Jang KL, Osada H, Jung M, Kim BY and Ahn JS: Modulation of e-cadherin expression by k-ras; involvement of DNA methyltransferase-3b. Carcinogenesis 31(7): 1194-1201, 2010. PMID: 20375073. DOI: 10.1093/carcin/bgq071

28 Tsunoda T, Ishikura S. DOI K, Iwaihara Y, Hidesima H, Luo H, Hirose Y and Shirasawa S: Establishment of a three-dimensional floating cell culture system for screening drugs targeting krasmediated signaling molecules. Anticancer Res 35(8): 4453-4459, 2015. PMID: 26168486.

29 Shirasawa S, Furuse M, Yokoyama N and Sasazuki T: Altered growth of human colon cancer cell lines disrupted at activated ki-ras. Science 260(5104): 85-88, 1993. PMID: 8465203. DOI: $10.1126 /$ science. 8465203

30 Nishi K, Iwaihara Y, Tsunoda T. DOI K, Sakata T, Shirasawa S and Ishikura $\mathrm{S}$ : Ros-induced cleavage of nhlrc2 by caspase- 8 leads to apoptotic cell death in the het116 human colon cancer cell line. Cell Death Dis 8(12): 3218, 2017. PMID: 29242562. DOI: $10.1038 / \mathrm{s} 41419-017-0006-7$

31 Ota T. DOI K, Fujimoto T, Tanaka Y, Ogawa M, Matsuzaki H, Kuroki M, Miyamoto S, Shirasawa S and Tsunoda T: Kras upregulates the expression of mir-181a, mir-200c and mir-210 in a three-dimensional-specific manner in dld-1 colorectal cancer cells. Anticancer Res 32(6): 2271-2275, 2012. PMID: 22641662.

32 Yoshida Y, Tsunoda T. DOI K, Fujimoto T, Tanaka Y, Ota T, Ogawa M, Matsuzaki H, Kuroki M, Iwasaki A and Shirasawa S: Alpk2 is crucial for luminal apoptosis and DNA repair-related gene expression in a three-dimensional colonic-crypt model. Anticancer Res 32(6): 2301-2308, 2012. PMID: 22641666.

33 Semenza GL: Regulation of cancer cell metabolism by hypoxiainducible factor 1. Semin Cancer Biol 19(1): 12-16, 2009. PMID: 21785006. DOI: 10.1016/j.semcancer.2008.11.009

34 Pugh CW, Tan CC, Jones RW and Ratcliffe PJ: Functional analysis of an oxygen-regulated transcriptional enhancer lying 3' to the mouse erythropoietin gene. Proc Natl Acad Sci USA 88(23): 1055310557, 1991. PMID: 1961720. DOI: 10.1073/pnas.88.23.10553

35 Riffle S, Pandey RN, Albert M and Hegde RS: Linking hypoxia, DNA damage and proliferation in multicellular tumor spheroids. BMC Cancer 17(1): 338, 2017. PMID: 28521819. DOI: 10.1186/s12885-017-3319-0

36 Huang RY, Wong MK, Tan TZ, Kuay KT, Ng AH, Chung VY, Chu YS, Matsumura N, Lai HC, Lee YF, Sim WJ, Chai C, Pietschmann E, Mori S, Low JJ, Choolani M and Thiery JP: An emt spectrum defines an anoikis-resistant and spheroidogenic intermediate mesenchymal state that is sensitive to e-cadherin restoration by a src-kinase inhibitor, saracatinib (azd0530). Cell Death Dis 4: e915, 2013. PMID: 24201814. DOI: 10.1038/cddis.2013.442

37 Depping R, von Fallois M, Landesman Y and Kosyna FK: The nuclear export inhibitor selinexor inhibits hypoxia signaling pathways and $3 \mathrm{~d}$ spheroid growth of cancer cells. Onco Targets Ther 12: 8387-8399, 2019. PMID: 31632086. DOI: 10.2147/ott.s213208

Received May 23, 2020

Revised June 5, 2020 Accepted June 8, 2020 